Central Washington University

ScholarWorks@CWU

All Faculty Scholarship for the College of the Sciences

2-15-1994

Wannier-Stark localization in modulation-doped multiplequantum-well structures

Roger H. Yu

Follow this and additional works at: https://digitalcommons.cwu.edu/cotsfac

Part of the Quantum Physics Commons 


\title{
Wannier-Stark localization in modulation-doped multiple-quantum-well structures
}

\author{
Roger H. Yu \\ Department of Physics, Central Washington University, Ellensburg, Washington 98926
}

(Received 12 July 1993; revised manuscript received 20 September 1993)

\begin{abstract}
The external-electric-field effect and the doping effect on a modulation-doped finite-length $\mathrm{GaAs} / \mathrm{Al}_{x} \mathrm{Ga}_{1-x} \mathrm{As}$ superlattice have been studied by solving the Schrödinger-Kohn-Sham equation and the Poisson equation self-consistently. In the heavy-doping case, the external electric field makes electrons sequentially tunnel through several potential barriers, and accumulates electrons near one side of the finite-length superlattice. The collective motion of the electrons results in high- and low-field domains in the superlattice. We have found that Wannier-Stark localization exists only in the high-field domain, and that the electronic states are not evenly spaced. The electric-field-induced (doping induced) depopulation (population) of subbands has been studied. We have also found oscillatory behavior of the calculated conductivity near the Fermi energy as a function of the external electric field.
\end{abstract}

A substantial electric field applied across a periodic potential associated with an infinitely long superlattice will lift the degeneracy of the electronic states found in minibands formed by the Bloch states. ${ }^{1}$ The solution of the one-electron Schrödinger equation associated with the tilted periodic potential, namely, the continuous energy spectrum, evolves into discrete and evenly spaced energy levels, $E_{n}=E_{f}+E_{0}$, forming the Stark ladder. ${ }^{1}$ The extended miniband states gradually become spatially localized (the so-called Wannier-Stark localization ${ }^{1}$ ) with the increase in the external electric field. The Wannier-Stark localizations in an undoped semiconductor superlattice have been verified by Mendez, Agullo-Rueda, and Hong, ${ }^{2}$ and Voisin et al. ${ }^{3}$ using optical-absorption techniques. Since then, photoluminescence and photocurrent spectroscopies have been employed to study the resonanceinduced delocalization of electronic states in superlattices, ${ }^{4,5}$ and the interactions between Stark ladder states and surface states. $^{6}$ The interaction between the embedded-quantum-well localized states, ${ }^{7}$ and the Wannier-Stark localization in a strained superlattice ${ }^{8}$ were also studied. Most recently, Feldmann et al. ${ }^{9}$ have performed a transient degenerate four-wave mixing (DFWM) experiment to study the optical dephasing of Stark ladder excitations in a semiconductor superlattice. The DFWM signal exhibits a periodic modulation with a time period corresponding to the energy spacing between the Stark ladder states. ${ }^{9}$

On the theoretical side, there have been a number of calculations based on the tight-binding approximation providing evidence of the existence of the WannierStark localization in a multiple-quantum-well structure. $^{2,4,6,10-12}$ The vector potential method ${ }^{13}$ and the numerical three-point derivative formula ${ }^{14}$ have also been used to solve the one-electron Schrödinger equation. A highly accurate and simple calculation scheme, via the Fourier series expansion, has been developed by Glessner and the present author. ${ }^{15}$ We considered an undoped finite-length superlattice with two bound minibands making the interaction between two Stark ladders possible. This is of particular interest since it is reminescent of the interband coupling in a bulk crystal. ${ }^{16}$ We have reported on the Wannier-Stark localization, the couplings of Tamm states and Stark ladders, and the delocalization effects resulting from mode mixing, as well as on anticrossing phenomena. ${ }^{15}$ To the best of our knowledge, all theoretical studies on the Wannier-Stark localization are based on the same footing: that electrons are independent and can be treated by the one-electron Schrödinger equation. While our solutions of the oneelectron Schrödinger equation undoubtedly show the Wannier-Stark localization in an undoped superlattice, ${ }^{15}$ we challenged ourselves by asking the following question: Does the Wannier-Stark localization still exist in a modulation-doped finite-length superlattice (MDFLS), in which many electrons are present? We anticipated that a self-consistent calculation ${ }^{17,18}$ would be required because strong interactions (mainly the Coulomb interaction) are expected in the many-electron system. For a zero electric field, we have introduced a nonuniform jellium model which incorporates alternating square-wave-like donor distribution in a modulation-doped $\mathrm{GaAs} / \mathrm{Al}_{x} \mathrm{Ga}_{1-x} \mathrm{As}$ superlattice $e^{17,18}$ to calculate the electronic structure. This approach is the first step toward a solution which includes crystallinity effects in the calculation of manyelectron dynamics.

In this paper, we will present the results of our selfconsistent calculations for the Wannier-Stark localization in a MDFLS exposed to an external electric field in the $(z)$ direction of growth. Figures 1(a) and 1(b) display an ionized donor distribution (nonuniform jellium model) and a square-wave-like potential profile, respectively, by dotted lines. The model has $N$ GaAs wells of width $w$, alternating with $N-1$ doped $\mathrm{Al}_{x} \mathrm{Ga}_{1-x}$ As barriers of width $b$ and, in addition, two undoped surface $\mathrm{Al}_{x} \mathrm{Ga}_{1-x}$ As barriers of width $d_{s}$ (Fig. 1 only shows the outermost three periods of the sample). The surface barrier height $E_{s}$ is different from the interior barrier height $E_{b}$ in general. Because the length of the electron tunneling into the vacuum is small compared to the period, two hard walls are assumed on both sides of the slab for the convenience of 
calculations. Within the framework of the effective-mass approximation, the ground-state charge density, discrete energy levels, and the Fermi energy for the interacting inhomogeneous electron gas can be well described by the self-consistent solution of the Schrödinger-Kohn-Sham and Poisson equations ${ }^{19}$ associated with the effective potential $v_{\text {eff }}$, which constitutes (1) the Hartree potential $v_{H}(z)$ (Ref. 17), (2) the exchange and correlation potential $v_{x c}(z)$ of Hedin and Lundqvist, ${ }^{20}$ (3) the background potential profile $E_{b}(z)$, and (4) the potential energy due to the external electric field $F, v_{F}(z)=-F e(z-L / 2)$, with the zero potential energy chosen at the center of the superlattice. Because electrons in a modulation-doped superlattice can tunnel sequentially through several potential barriers, as shown by the experiment, ${ }^{21}$ we have used the condition of overall-sample charge neutrality to calculate the Fermi energy self-consistently. In our case, the assumption of charge neutrality for each period ${ }^{22}$ should be avoided. In our self-consistent calculations, sine functions are used to expand the wave functions $\phi_{l}(z)$ and the effective potential $v_{\text {eff }}(z)$ in discretizing the SchrödingerKohn-Sham equation. The details of our calculations can be found in our earlier work. ${ }^{15,17,18}$

The solid lines in Figs. 1(a) and 1(b) present the selfconsistent charge density and the effective potential at zero external electric field for a superlattice of 11 periods $(N=11)$, with $d_{s}=100 \AA, w=80 \AA, b=20 \AA$, and

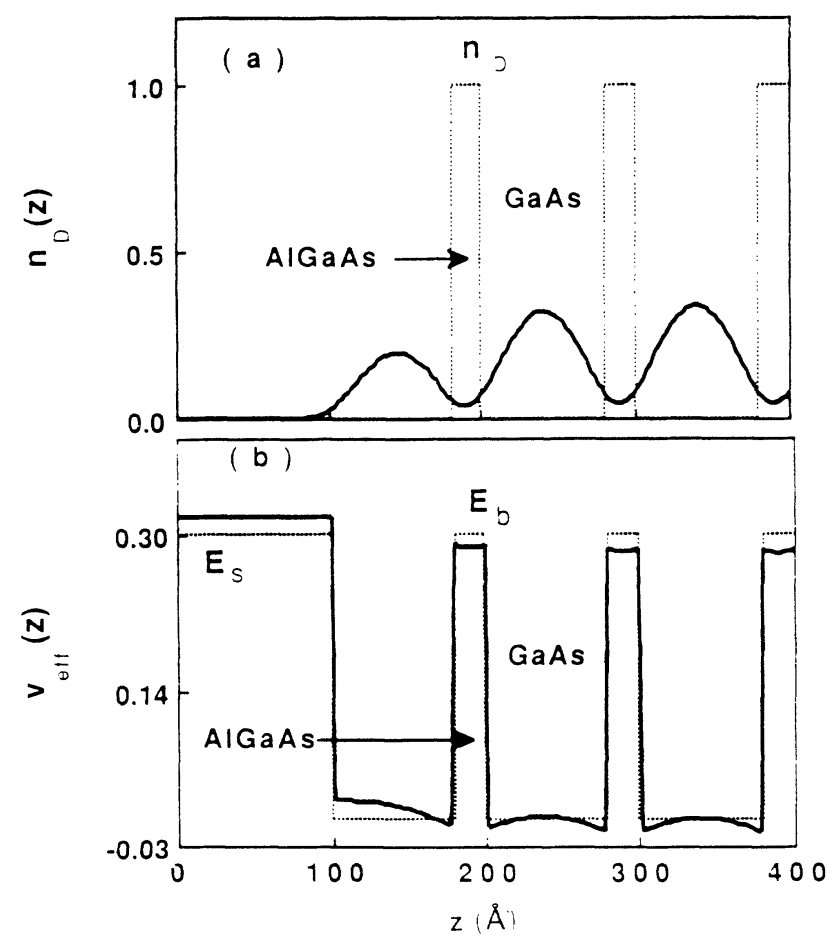

FIG. 1. (a) and (b) show the potential background and doping profile (dotted lines), and the self-consistent charge density and effective potential (solid lines), at zero electric field, for an 11period superlattice with $d_{s}=100 \AA, w=80 \AA, b=20 \AA$, and $E_{b}=E_{s}=0.3 \mathrm{eV}$. The charge density is given in the unit of doping density $n_{D}=5.0 \times 10^{-6} \AA^{-3}$.
$E_{b}=0.3 \mathrm{eV}$, and with a (heavy) doping density $n_{D}=5.0 \times 10^{-6} \AA^{-3}$. We have shown that a nonzero difference between the surface and interior barrier heights $\left(\Delta E=E_{s}-E_{b}=0\right)$ yields surface-localized Tamm states. ${ }^{15,17,18}$ For clarity and present interest, we have assumed equal surface and interior barriers to avoid surface states in the calculated band structure. The charge density in the outermost well, shown in Fig. 1, is slightly lower than that of the interior wells. Since the potential barrier is relatively low, the electron tunneling results in a fairly high charge density at the doping sites. The inhomogeneity of the electron gas in the superlattice causes different local electric fields in the wells and barriers; furthermore, it bends the conduction-band edge of the wells (barriers) upward (downward) as exhibited by the solid lines in Fig. 1(b). The self-consistent calculation of the band structure for light doping, $n_{D}=1.0 \times 10^{-8} \AA^{-3}$, was also carried out using the same parameters otherwise. Band structures for different doping densities are similar for zero external electric field: that is, there are two bound minibands of widths $\Delta_{1}=0.038 \mathrm{eV}$ and $\Delta_{2}=0.084$ $\mathrm{eV}$ centered at 0.045 and $0.176 \mathrm{eV}$, respectively. The main difference between heavy and light doping is the Fermi energy: for the former $E_{F}=0.075 \mathrm{eV}$, with 11 eigenstates occupied; for the latter the Fermi energy is slightly above the lowest energy level. For the lightly doped superlattice, the conduction-band edge is almost flat (like a step function) due to the weakness of the local electric fields produced by the inhomogeneous charge distribution. Figures 2 (a) and 2(b) display the energy levels vs the external electric field $F$ for light $\left(n_{D}=1.0 \times 10^{-8}\right.$ $\left.\AA^{-3}\right)$ and heavy $\left(n_{D}=5.0 \times 10^{-6} \AA^{-3}\right)$ doping, respectively. The light-doping results mimic the double-fan diagram (two Stark ladders) we have obtained ${ }^{15}$ for an undoped superlattice by solving the one-electron Schrödinger equation. The two minibands below the potential barrier evolve first nonlinearly and then linearly into evenly spaced Stark ladder states as $F$ increases. Anticrossings between the two Stark ladders occur when $F$ is greater than $13 \mathrm{kV} / \mathrm{cm}$. At this electric field the top state (confined in the extreme left well) of the lower Stark ladder, and the bottom state (localized in the rightmost well) of the higher Stark ladder, are aligned in energy. However, their corresponding wave functions are so far displaced in space that the repulsive interaction is weak, and the two modes seem to cross each other at $F=13$ $\mathrm{kV} / \mathrm{cm}$. At about the same electric-field strength, the continuous states (above the barrier) begin to anticross the higher Stark ladder states. A certain lower Stark ladder state varies parallel to its counterpart in the higher Stark ladder, since every quantum well preserves energy separations as $F$ increases. The energy spacing in the lower Stark ladder $\left(\Delta_{1} / 10+e F d\right)$ is slightly smaller than that in the higher Stark ladder $\left(\Delta_{2} / 10+e F d\right)$. At zero electric field the band gap between the two bound minibands is $\left(0.176 \mathrm{eV}-\Delta_{2} / 2\right)-\left(0.045 \mathrm{eV}-\Delta_{1} / 2\right)=0.07 \mathrm{eV}$. When an external electric field is applied, the band structure is destroyed, and the energy gap increases to the difference between two eigenstates of an isolated quantum well, $0.176-0.045 \mathrm{eV}=0.131 \mathrm{eV}$. This increase in energy is the blueshift of the optical-absorption edge discussed 


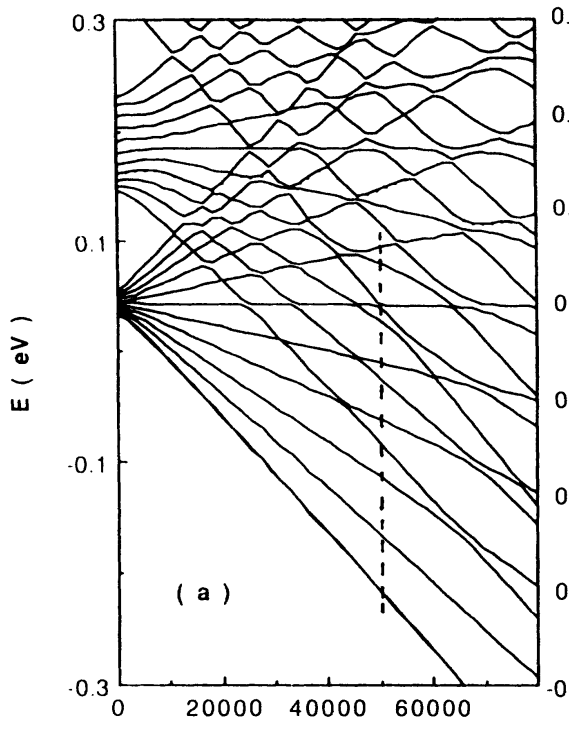

$\mathrm{F}(\mathrm{V} / \mathrm{cm})$

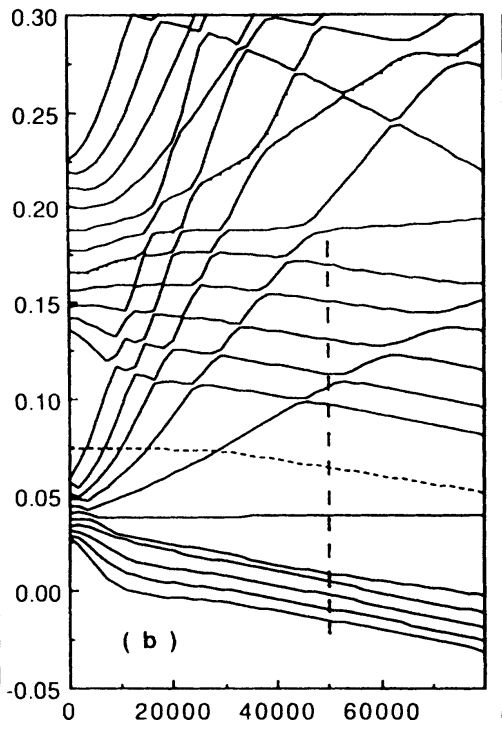

$F(\mathrm{~V} / \mathrm{cm})$

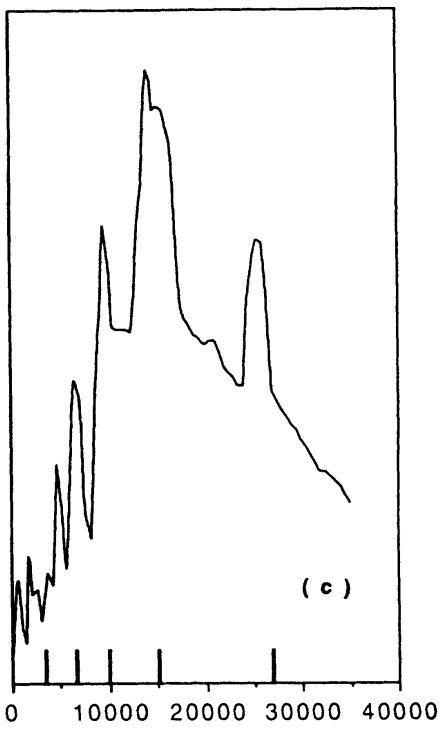

$F(\mathrm{~V} / \mathrm{cm})$

FIG. 2. (a) and (b) display the energy vs electric-field $F$ diagram, the fan diagram, for light $\left(n_{D}=1.0 \times 10^{-8} \AA^{-3}\right)$ and heavy $\left(n_{D}=5.0 \times 10^{-6} \AA^{-3}\right)$ doping densities, respectively. The other parameters are the same as given in Fig. 1. The Fermi energy is shown by the dashed line. (c) Calculated dynamical conductivity given in arbitrary units. The parameters used in the calculation are the same as in (b).

by Bleuse, Bastard, and Voisin. ${ }^{10}$

For the heavily doped superlattice, the fan diagram is strongly suppressed upward in energy, as shown in Fig. 2(b). At weak electric field (smaller than $10 \mathrm{kV} / \mathrm{cm}$ ), both minibands diverge asymmetrically with respect to their centers; the slope of the highest state is greater in magnitude than that of the lowest one, which, in turn, gives rise to an unevenly spaced energy spectrum. This asymmetry is a signature of the sequential tunneling of electrons through the barriers. At the critical electric field $\left(F_{c}=10\right.$ $\mathrm{kV} / \mathrm{cm}$ ) and beyond, the majority of the electrons accumulate in the right half of the superlattice, inducing a new miniband formed by the lowest five eigenstates. As a result of the external electric field, electrons tunnel toward the right half of the sample, and the eigenstates localized in the wells of the left half of the superlattice are depopulated. The electric-field-induced energy-levelemptying effect reminds us of the Shubnikov-de Haas effect: the magnetoresistivity oscillates as the external magnetic field increases because of the consecutive Landau-level depopulation, and near the Fermi energy the density of states exhibits an oscillatory behavior which is periodic in $B^{-1}$. We have observed this electric-field-dependent change in the number of occupied states. The density of states also changes in a stepwise manner whenever the Fermi energy [the dashed line in Fig. 2(b)] crosses an energy level as the external electric field increases. It is expected that the electric-fieldinduced change in the number of occupied states and in the density of states near the Fermi energy will result in a modulation in the conductivity. We have calculated the dynamical conductivity at the Fermi energy $\sigma\left(\omega=E_{F}\right)$ using the dynamical polarizability $\alpha\left(\omega=E_{F}\right)$ through the following equation: ${ }^{23}$

$$
\sigma\left(\omega=E_{F}\right)=\omega \operatorname{Im}\left[\alpha\left(\omega=E_{F}\right)\right] .
$$

The dynamical response of the inhomogeneous electron system is described by the density-density correlation function $\chi\left(q_{\|}, \omega ; z, z^{\prime}\right)$ which can be obtained by solving a Dyson-type integral equation:

$$
\begin{aligned}
\chi\left(q_{\|}, \omega ; z, z^{\prime}\right)= & \chi^{0}\left(q_{\|}, \omega ; z, z^{\prime}\right) \\
& +\int_{0}^{L} \int_{0}^{L} d z_{1} d z_{2} \chi^{0}\left(q_{\|}, \omega ; z, z_{1}\right) V\left(q_{\|}, z_{1}, z_{2}\right) \\
& \times \chi\left(q_{\|}, \omega ; z^{\prime}, z_{2}\right),
\end{aligned}
$$

where $\chi^{0}\left(q_{\|}, \omega ; z, z^{\prime}\right)$ is the response function of the noninteracting electron system, and $V$ is the Fourier component of the electron-electron interaction potential. ${ }^{18}$ The dynamical polarizability $\alpha\left(\omega=E_{F}\right)$ is related to the response function $\chi$ through the equation ${ }^{24}$

$$
\begin{aligned}
\alpha\left(\omega=E_{F}\right)=\int_{0}^{L} \int_{0}^{L} d z d z^{\prime} \exp \left[-\lambda\left(z+z^{\prime}\right)\right] \\
\\
\times \chi\left(q_{\|} \rightarrow 0, \omega=E_{F} ; z, z^{\prime}\right),
\end{aligned}
$$

in which an exponentially decaying external driving field $V_{\text {ext }} \sim e^{-\lambda z-i \omega t}$ was assumed. The calculated conductivity $\sigma\left(E_{F}\right)$, shown in Fig. 2(c), displays strong oscillations as the external electric field increases. The electric fields at which the depopulation of subbands occurs are marked by short vertical lines. The oscillation in the conductivity is not periodic in the inverse of the external electric field, $F^{-1}$, although the electric-field difference corresponding to the consecutive energy level emptying is increasing. For a doped finite-length superlattice, the ideal conditions for $F^{-1}$ periodicity in the subband depopulation do not exist, since neither an evenly spaced energy spectrum nor a zero bandwidth at $F=0$ is satisfied. Absorption 
spectra can also be used to probe the change in the density of states induced by the external electric field by tuning the photon energy close to the interband resonance. The absorption coefficient changes whenever an energy level crosses the fixed photon energy. ${ }^{10}$ For a weaker electric field $\left(F<F_{c}\right)$, in addition to the band bending introduced by the inhomogeneity of the electron gas, the envelope of the potential profile is also bent downward parabolically because of the immigration of the electrons to the right side of the superlattice. For strong electric fields $F>F_{c}$, the overall potential profile can be divided into high- and low-field domains: in the former, the wave functions are localized, while in the latter they are extended constituting a new miniband. If the external electric field is still stronger, $F>40 \mathrm{kV} / \mathrm{cm}$, the low-field domain supports a second miniband, as shown in Fig. 2(b). Thus, in a modulation-doped finite-length superlattice, the Wannier-Stark localization still exists, but only in the high-field domain.

To better understand the Wannier-Stark localization and the formation of new minibands, in Figs. 3(a) and 3 (b) we depict the lowest 12 squared wave functions [see all the states intersected by the dashed vertical lines in Figs. 2(a) and 2(b)] in the order of their corresponding eigenenergies from bottom to top for low and high doping densities, respectively. For the low doping case, all the wave functions with one peak (evolved from the lowest miniband at $F=0$ ) are highly localized in a quantum well, and the Stark ladder states originating from the second miniband have a mode in each well, as expected, and extend slightly into adjacent wells. It is seen in Fig. 2(a) that for $F=50 \mathrm{kV} / \mathrm{cm}$ the tenth state emerges from the above-barrier continuous states. Actually the state is strongly localized above the extreme right surface barrier, as has been observed experimentally. ${ }^{25}$ In Fig. 2(a), near $F=50 \mathrm{kV} / \mathrm{cm}$ and $E=0.04 \mathrm{eV}$, three eigenstates, two Stark ladder states, and one above-barrier state are aligned in energy, giving rise to a triplet resonant interaction. Such an interaction between two states should result in linearly combined even and odd resultant wave functions, and the eighth, ninth, and tenth states in Fig. 3(a) show that indeed their wave functions are linear combinations of the states without interaction. The selfconsistent charge density and potential profile for the heavy doping, $n_{D}=5.0 \times 10^{-6} \AA^{-3}$ and $F=50 \mathrm{kV} / \mathrm{cm}$, are shown in Fig. 3(b). Because of the sequential tunneling of many electrons toward the right side of the model in the steady state, the external electric field experienced by electrons is almost zero in the region of electron accumulation, while the effective electric field in the region of electron depletion doubles the external electric field, $F_{\text {effective }}=\Delta V / \Delta L \sim 0.5 \mathrm{eV} / 500 \AA=100 \mathrm{kV} / \mathrm{cm}$. This effect is absent in the light-doping case since the immigration of electrons does not affect the external electric field significantly. The potential drop would cover the whole sample instead of a shorter distance, producing a stronger effective electric field. The lowest 12 squared wave functions shown in Fig. 3(b) demonstrate both new miniband states and localized states. The sixth state is confined in the middle quantum well, and it separates the occupied miniband from the empty one. The twelfth state and beyond (not shown here) are basically confined in the wells in the left half of the superlattice.

The formation of new minibands due to the manyelectron effect is further illustrated by the energy versus doping density curve in Fig. 4. For the doping density $n_{D}=1.0 \times 10^{-8} \AA^{-3}$, the diagram is reduced to a vertical line in Fig. 2(a) for $F=50 \mathrm{kV} / \mathrm{cm}$. When the doping density $n_{D}$ increases, more states penetrate the Fermi energy $E_{F}$ as shown by the dashed line. As a result of the charge accumulation in the right half of the superlattice, the envelope of the potential profile is strongly affected by the

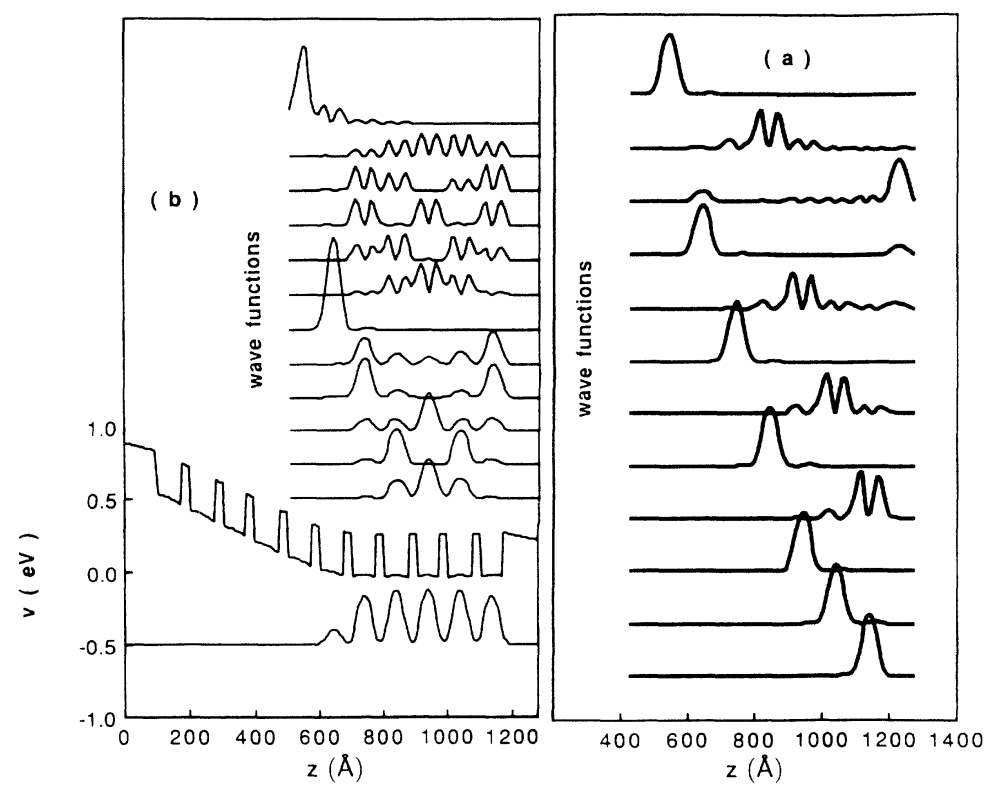

FIG. 3. (a) and (b) show the lowest 12 selfconsistently calculated squared-wave functions for the cases of low doping density, $n_{D}=1.0 \times 10^{-8} \AA^{-3}$ and high doping density, $n_{D}=5.0 \times 10^{-6} \AA^{-3}$, respectively, at $F=50$ $\mathrm{kV} / \mathrm{cm}$. The bottom panel of (b) represents the self-consistent charge density and effective potential. 


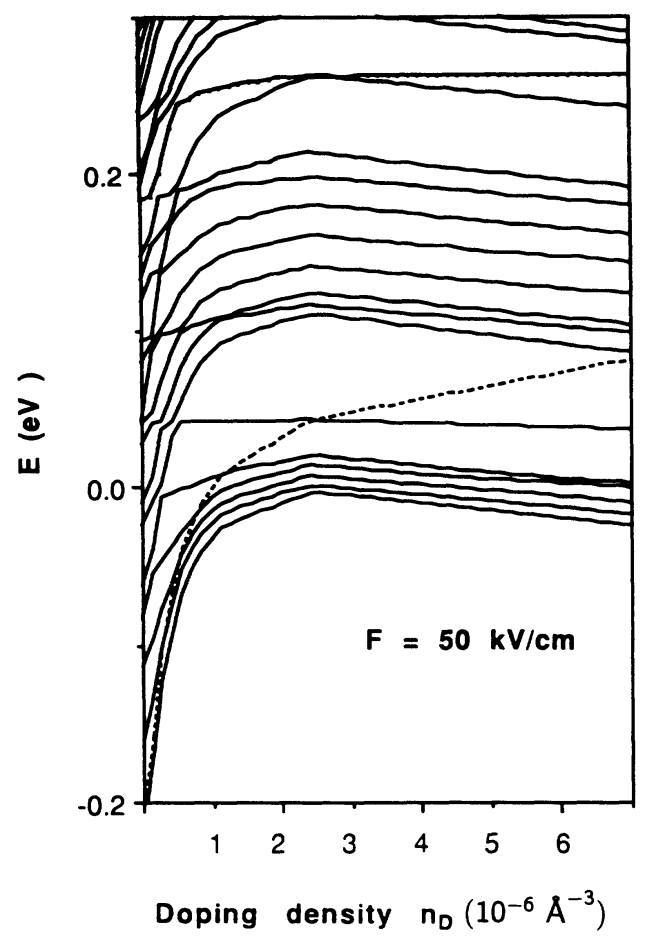

FIG. 4. Energy vs doping density for $F=50 \mathrm{kV} / \mathrm{cm}$ and the other parameters are taken from Fig. 1. The dashed line represents the Fermi energy.

local electric field, making it less tilted (low-field domain); therefore, the energy levels corresponding to the states initially confined in the quantum wells near the right side of the superlattice are elevated in energy. At the critical doping density $2.5 \times 10^{-6} \AA^{-3}$, the saturated potential profile supports two minibands in the low-field domain. When the doping density becomes still larger, the Fermi energy increases while the lowest five energy levels decrease in a linear fashion. The higher new miniband begins to be populated when $n_{D}$ is greater than $7.0 \times 10^{-6}$ $\AA^{-3}$; however, the shape of the potential remains essentially unchanged. At a given external electric field, the density of states near the Fermi energy jumps whenever a new energy level is filled as a result of an increase in doping density.

In conclusion, we have found exact solutions to the Poisson and Schrödinger equations self-consistently for a modulation-doped finite-length superlattice exposed to an external electric field along the growth direction. The electronic level structure and Wannier-Stark localization under the influence of an external electric field and given doping density, and the many-electron effect, are discussed in detail. The external electric field first tilts the conduction-band edge of the superlattice and localizes the wave functions, then it redistributes the electrons until the electronic system has become stabilized. For a fixed heavy-doping density, the external electric field pulls electrons, sequentially tunneling them through several quantum barriers and accumulating them near the right side of the sample. As the external electric field increases in magnitude, the local electric field due to the accumulation of electrons balances the external electric field, and hence divides the superlattice into high- and low-field domain. In the latter new minibands are introduced, and in the former the wave functions are confined. On the other hand, for a given external electric field, an increase in doping density also yields a new miniband. When the applied electric field (doping density) becomes stronger, the number of occupied states decreases (increases), and the new emptying (filling) effect causes a sudden drop (jump) in the density of states near the Fermi energy. We have predicted the oscillatory dynamical conductivity as a function of the external electric field; an effect that mimics the Shubnikov-de Haas oscillation in magnetoconductivity. The sample parameters we have used in the study are chosen in such a way that there are two minibands below the potential barrier, with only the lower one occupied. The system investigated here also provides insight into the interband transition of a bulk semiconductor crystal.

The author gratefully acknowledges the financial support of the Central Washington University Foundation.
${ }^{1}$ G. H. Wannier, Elementary Solid State Theory (Cambridge University Press, London, 1965).

${ }^{2}$ E. E. Mendez, F. Agullo-Rueda, and J. M. Hong, Phys. Rev. Lett. 60, 2426 (1988).

${ }^{3}$ P. Voisin, J. Bleuse, C. Bouche, S. Gaillard, and C. A. Alibert, Phys. Rev. Lett. 61, 1639 (1988).

${ }^{4}$ H. Schneider, H. T. Grahn, K. v. Klitzing, and K. Ploog, Phys. Rev. Lett. 65, 2720 (1990).

${ }^{5}$ M. Nakayama, I. Tanaka, H. Nishimura, K. Lawashima, and K. Fujiwara, Phys. Rev. B 44, 5935 (1991).

${ }^{6}$ F. Agullo-Rueda, E. E. Mendez, H. Ohno, and J. M. Hong, Phys. Rev. B 42, 1470 (1990).

${ }^{7}$ R. P. Leavitt and J. W. Little, Phys. Rev. B 41, 5174 (1990).

${ }^{8}$ B. Soucail, N. Dupuis, R. Ferreira, P. Voisin, A. P. Roth, D. Morris, K. Gibb, and C. Lacelle, Phys. Rev. B 41, 8568 (1990).

${ }^{9}$ J. Feldmann, K. Leo, J. Shah, D. A. B. Miller, J. E. Cunning- ham, T. Meier, G. von Plessen, A. Schulze, P. Thomas, and S. Schmitt-Rink, Phys. Rev. B 46, 7252 (1992).

${ }^{10} \mathrm{~J}$. Bleuse, G. Bastard, and P. Voisin, Phys. Rev. Lett. 60, 220 (1988).

${ }^{11}$ D. Emini and C. F. Hart, Phys. Rev. B 36, 7353 (1987).

${ }^{12}$ J. Leo and A. MacKinnon, J. Phys. Condens. Matter 1, 1449 (1989).

${ }^{13}$ J. B. Krieger and G. J. Iafrate, Phys. Rev. B 33, 549 (1986).

${ }^{14}$ O. Gulseren and S. Ciraci, Phys. Rev. B 46, 7621 (1992).

${ }^{15}$ William Glessner and Roger Yu (unpublished).

${ }^{16}$ H. Schneider, K. Kawashima, and K. Fujiwara, Phys. Rev. B 44, 5943 (1991).

${ }^{17}$ Roger H. Yu, Phys. Rev. B 47, 15692 (1993).

${ }^{18}$ Roger H. Yu, Phys. Rev. B 47, 1379 (1993).

${ }^{19}$ W. Kohn and L. J. Sham, Phys. Rev. 140, A1133 (1965).

${ }^{20}$ L. Hedin and B. I. Lundqvist, J. Phys. C 4, 2064 (1971).

${ }^{21}$ K. K. Choi, B. F. Levine, R. J. Malik, J. Walker, and C. G. 
Bethea, Phys. Rev. B 35, 4172 (1987).

${ }^{22}$ B. Jogai, M. O. Manasreh, C. E. Stutz, P. L. Whitney, and D. K. Kinell, Phys. Rev. B 46, 7208 (1992); M. O. Manasreh, B. Jogai, C. E. Stutz, and D. C. Reynolds, J. Appl. Phys. 73, 3105 (1993).

${ }^{23}$ F. Stern, Phys. Rev. Lett. 18, 548 (1967); T. Ando, A. B. Fowler, and F. Stern, Rev. Mod. Phys. 54, 437 (1982), and references therein.

${ }^{24}$ A. Rubio, L. C. Balbas, and J. A. Alonso, Phys. Rev. B 46, 4891 (1992).

${ }^{25}$ F. C. Zhang, N. Oai, H. Luo, N. Samarth, M. Dobrowolska, J. K. Furdyna, and L. K. Ram-Mohan, Phys. Rev. Lett. 68, 3220 (1992). 